\title{
Publisher Correction: Role of the BUB3 protein in phragmoplast microtubule reorganization during cytokinesis
}

Hongchang Zhang, Xingguang Deng, Baojuan Sun, Sonny Lee Van, Zhensheng Kang, Honghui Lin, Yuh-Ru Julie Lee (1) and Bo Liu (D)

Correction to: Nature Plants https://doi.org/10.1038/s41477-018-0192-z, published online 2 July 2018.

In the version of this Article originally published, the affiliation for author Yuh-Ru Julie Lee was incorrect; the correct affiliation is '2Department of Plant Biology, College of Biological Sciences, University of California, Davis, CA, USA'. This has now been amended in all versions of the Article. 